\title{
Using a therapeutic companion robot for dementia symptoms in long-term care: reflections from a cluster-RCT
}

\begin{abstract}
Objectives: We undertook a cluster-randomised controlled trial exploring the effect of a therapeutic companion robot (PARO) compared to a look-alike plush toy and usual care on dementia symptoms of long-term care residents. Complementing the reported quantitative primary outcomes from the trial, this paper aims to provide critical reflection and commentary on individual participant responses to PARO, observed through video recordings during the trial, with a view to informing clinical practice and research.
\end{abstract}

Method: A descriptive, qualitative design was employed, with five participants selected from the PARO intervention arm of the trial to permit discussion of individual responses to PARO during the 10-week trial. The trial is registered with the Australian New Zealand Clinical Trials Registry (ACTRN12614000508673).

Results: The five participants and their responses to PARO are presented in terms of three issues: i.) Different pre-intervention clinical presentations and different responses; ii.) Same individual, different response - the need for continual assessment and review; and iii.) The ethics of giving and retrieving PARO. Implications for clinical practice and future research are discussed in relation to each issue.

Conclusion: The findings of this qualitative study suggest that one approach does not fit all, and that there is considerable variation in responses to PARO. A number of recommendations are discussed to aid the delivery of psychosocial interventions with PARO in practice, as well as to guide future research. 
Keywords: Cognitive Impairment, Care Planning, Psychosocial Interventions, Qualitative, Robotics 


\section{Using a therapeutic companion robot for dementia symptoms in long-term care: reflections from a cluster-RCT}

\section{Introduction}

Between 50 to $80 \%$ of residents in long-term care (LTC) facilities have dementia (Alzheimer's Association, 2016; Australian Institute of Health and Welfare, 2012b; Gaugler, Yu, Davila \& Shippee, 2014; Prince et al., 2014) and, of these, as many as four-fifths are affected by adverse neuropsychiatric symptoms (Australian Institute of Health and Welfare, 2012a; Zuidema, Derksen, Verhey \& Koopmans, 2007). Behavioural and psychological symptoms of dementia (BPSD), which includes disturbed perception and thought content, frequent mood changes, and agitated behaviour (Finkel \& Burns, 1999), can be distressing for both the person with dementia and the carer. For the person with dementia, quality of life and functionality can be reduced (Klapwijk, Caljouw, Pieper, van der Steen \& Achterberg, 2016), whilst for carers, BPSD can make the task of caregiving stressful and dissatisfying (Gaugler et al., 2010; Porter et al., 2016; Zimmerman et al., 2005). Historically, psychotropic medication has been prescribed as the first choice of treatment to ameliorate BPSD. However, given the many known side-effects (Seitz et al., 2013), inconclusive treatment efficacy (Seitz et al., 2013), and high rate of inappropriate prescription of medication (Moyle et al., 2017a; van der Spek et al., 2016), psychosocial interventions have been advocated as the first-line treatment for managing BPSD (Livingston et al., 2014; Oyebode \& Parveen, 2016).

Of the many psychosocial interventions available, PARO (version 9; Figure 1), a therapeutic pet-type robot modelled on the features of a baby harp seal, has shown potential to ease BPSD in the few, small-scale clinical trials conducted to date (Jøranson, Pedersen, Rokstad \& Ihlebæk, 2015; Moyle et al., 2013; Petersen, Houston, Qin, Tague \& Studley, 2017; Takayanagi, Kirita \& Shibata, 2014; Thodberg et al., 2016). Building on this initial 
work, and in response to calls for better quality trials in the area (Bemelmans, Gelderblom, Jonker \& de Witte, 2012; Mordoch, Osterreicher, Guse, Roger \& Thompson, 2013), we undertook a parallel, three-group cluster-randomised controlled trial (RCT) that compared PARO with a look-alike plush toy (PARO with artificial intelligence disabled) and usual care, on the psychological and behavioural symptoms of dementia for those living in LTC. The primary outcomes from the study were analysed quantitatively, with modest but statistical effects found, suggesting that the PARO intervention was an effective and feasible psychosocial intervention to help manage BPSD for some individuals with dementia (Moyle et al., 2017b).To complement these quantitative findings, this paper provides a critical reflection and commentary on the potential heterogeneity in responses to the PARO during the 10-week trial. Incorporating qualitative methods within RCTs, although still relatively uncommon in dementia research, is recognised as an important means of situating the effects within the context of the lived experience (Gibson, Timlin, Curran \& Wattis, 2004), as well as widening understanding beyond the statistically significant findings. Importantly, qualitative components also enable researchers to explore aspects of the psychosocial intervention that work and do not work, for whom, and in what situations. Doing so has very real and practical implications for delivering the intervention in an 'everyday' context (Lawrence, Fossey, Ballard, Moniz-Cook \& Murray, 2012; Lewin, Glenton \& Oxman, 2009).

[Insert Figure 1 about here]

\section{Methods}

\section{Design}

A descriptive, qualitative design was employed, with participants selected from the PARO intervention arm of the cluster-RCT to permit critical reflection and discussion of individual 
responses to PARO during the 10 -week trial, with a view to informing clinical practice and future research.

\section{The PARO intervention group}

Between June 2014 and May 2015, a total of 415 participants from 28 LTC facilities in South-East Queensland, Australia were enrolled in this cluster-RCT. Participants were all aged 60 years or older and had a documented diagnosis of dementia. Exclusion criteria were: respite care admission; a dual diagnosis of a serious/persistent mental illness; terminal illness; and unremitting pain/distressing physical symptoms. Full details of the study protocol (Moyle et al., 2015) are reported elsewhere. The trial is registered with the Australian New Zealand Clinical Trials Registry (ACTRN12614000508673).

Within the PARO intervention arm of the cluster-RCT, there were 138 participants from nine LTC facilities. Intervention sessions involved individual, 15-min sessions with PARO (version 9) three afternoons a week, between 13:00 and 17:00, Monday, Wednesday, and Friday, for 10 weeks. All sessions were un-facilitated - the participant was given PARO by a trained Research Assistant (RA) and left to interact with it as they wished - and conducted wherever the participant happened to be at the time, unless in the bathroom. All RAs involved in the intervention arm were trained in the use of PARO, including the maintenance of it, such as charging the battery, as well as cleaning and infection control procedures. This included: the use of alcohol wipes for hand hygiene prior to any handling of PARO; the cleaning of PARO after each session using a disinfectant spray and disinfectant wipes; the cleaning of PARO’s storage container each week with disinfectant wipes; and the use of PARO prohibited with participants that had contact precautions and/or open wounds. Care staff were not required to assist in any aspect of the PARO intervention. 
Direct video recordings were made of participants for 30-mins, immediately before (15 min) and during (15 min) sessions with PARO at weeks 1, 5, and 10 by the same trained RA. All recorded video observations were coded by trained RAs, not involved in the intervention arm, in Noldus Observer XT® using the Video Coding Incorporating Observed Emotion Scheme (Jones, Sung \& Moyle, 2015).

A range of demographic data were collected for each participant at baseline, as well as information about each facility. Cognitive impairment was measured by trained RAs at baseline using the Rowland Universal Dementia Assessment Scale (RUDAS) (Rowland, Basic, Storey \& Conforti, 2006); a brief screening measure that minimises the effects of cultural learning and language diversity on assessment. Possible total scores of the RUDAS can range from 0-30, with lower scores indicative of greater cognitive impairment. Regarding baseline levels of agitation/behavioural disruption, facility care staff completed the 14-item proxy-version of the Cohen-Mansfield Agitation Inventory - Short Form (CMAI-SF) (Werner, Cohen-Mansfield, Koroknay \& Braun, 1994). Using a five-point scale, which ranged from 'never' to 'a few times an hour of continuous for half an hour or more', staff rated the frequency that each participant displayed 14 behaviours over the course of the previous two-week period. Possible total score of the CMAI-SF can range from 14-70, with higher scores indicative of greater agitation.

As we report elsewhere (Moyle et al., 2017b), at the start of the study, participants in the intervention group were similar in their demographic profile to those randomly allocated and participating in the plush toy and usual care comparison groups.

\section{Selection of participants}

We purposefully selected participants to permit critical reflection and commentary on individual responses to PARO during the 10 -week intervention. This selection process 
occurred post-hoc on completion of the cluster-RCT, and was a subjective process that evolved over the intervention period. First, during the trial, research team personnel including senior trial investigators, the Project Manager, Cluster Leaders, RAs involved in delivering the intervention, and RAs coding the recorded video data - identified noteworthy participant responses to PARO (i.e., positive, negative, unusual; unexpected; expected responses etc.). These responses were raised and discussed with the lead trial investigator (WM) as the intervention progressed (during regular project meetings and email correspondence), as a means of monitoring trial progress. Second, on trial completion, a smaller group of research team members (WM, CJ, \& JM) discussed the highlighted responses and, as a result, developed three issues that they felt had relevance to clinical practice and psychosocial intervention research. Third, demographic data collected at baseline were used to guide the identification of participants as potentially suitable examples of the issues. Fourth, the video observation data of potential participants was reviewed, with all their available observation data collected during the intervention at weeks 1,5 , and 10 viewed by one team member (JM). For all potential participants, details of their demographic profile and responses, observed via video, were recorded in a purpose-designed Microsoft Excel (2013) spreadsheet. Finally, the same group of team members who derived the issues (WM, CJ, \& JM), discussed and selected participants that they considered appropriate examples to permit critical reflection and discussion of the areas relevant to future clinical practice and psychosocial intervention research.

It should be noted that, whilst the team endeavoured to select participants with a range of demographic characteristics, the selection was issue-driven and, thus, profile diversity was not the primary focus. 


\section{Ethics}

Ethical approval for the study was provided by Griffith University Human Research Ethics Committee (NRS/03/14/HREC), and participating care organisations, as necessary. Written informed consent (from the person with dementia, if capable, or their next-of-kin) was obtained from all selected participants at the time of study enrolment, and separate written informed consent was also sought at enrolment for the future use of identifiable video data by the research team. Only participants for whom both written consents were obtained are discussed in this paper. However, to avoid unnecessary identification of residents, pseudonyms are used, and only general, cursive descriptions of demographic characteristics are provided.

\section{Results and discussion}

From the 138 participants who received the PARO intervention, we selected five participants who displayed exemplary responses to the three issues identified to critically discuss. Each issue is discussed in the following section in terms of their implications for clinical practice and future research with PARO. The baseline demographic and clinical profile of the five participants, and details of the facility they resided in, are provided in Table 1.

[Insert Table 1 about here]

\section{Issue 1: Different pre-intervention clinical presentations and different responses}

A person-centred approach to dementia care planning is essential, as different demographic and clinical profiles, unmet needs and interests and likes, can result in very different responses to the same psychosocial intervention. In the described examples, we present two residents from the same facility, who presented very differently immediately before the 
intervention, and subsequently responded in opposite ways to PARO; one positively and one negatively.

Participant 1 - Positive response to PARO: Annie was sitting alone at a table when PARO was introduced to her in a session in week 1 of the trial. PARO was placed facing forward on the table (face-to-face), and Annie instantly became engaged with it. During the first four minutes, she leaned forward in her chair and smiled, talked to PARO, stroked it, put her arm around it, and tickled it under its chin. She also pressed her forehead to PARO’s face a few times, remaining there for a number of seconds on each occasion. Annie remained passively engaged during the second third of the session (5-10 mins), sitting back in her chair, closing her eyes, and stroking PARO. During the last five minutes of the session, Annie fell asleep in her chair with PARO in front of her on the table. Annie responded in a similar way in other sessions she had with PARO, always showing a positive response. Annie appeared to like to hold PARO so that it was face-to-face with her during sessions, and she typically pressed her forehead on PARO’s face, or rested her head on it whilst holding it vertically on her chest.

Participant 2 - Negative response to PARO: Even before PARO was given to Margaret in week 1 of the intervention, she was agitated, engaging in a verbally aggressive, repetitive conversation with herself (as if another person were there), which culminated in her attempting to kick the RA when she came into her room and demand she leave her bedroom. After a few minutes, Margaret permitted the RA to come back into her bedroom, appearing as if the incident had not just occurred. When the RA attempted to give her PARO, Margaret was perched on the side of her bed, holding a piece of paper. She was verbally aggressive, standing up off the bed and saying ‘well I can’t because I’ve got to go and see my shoes’ and, in a raised voice with hand gestures, 'I don’t want it'. Margaret was very mobile, and was commonly walking up and down the facility corridor, and going into and out of her bedroom 
during all attempts to engage her in a session with PARO. In week 5, she refused to let the RA into her room even before PARO was shown, repeating, as she did in week 1, 'no, I have to go and see my shoes'. In week 10, whilst walking past PARO in the corridor, Margaret did stroke it under the chin as she passed. However, she was not interested in holding it when asked by the RA and did not actively look at PARO and, instead, continued to walk around the facility.

\section{Implications for practice}

Best practice guidelines recommend a person-centred approach to care planning and, when selecting the most appropriate psychosocial intervention for the individual, clinicians should work with the person with dementia, their families and immediate carers to identify the resident's needs, preferences, skills, and abilities (Alzheimer's Association, 2009; National Institute for Health and Care Excellence, 2006, updated 2016; NHMRC Partnership Centre for Dealing with Cognitive and Related Functional Decline in Older People, 2016). For psychosocial interventions involving PARO, it is important to understand the person's biography, particularly their like or dislike of animals, as this may be an initial indication of whether they may enjoy robotic animal-assisted therapies. Alongside this, it is also important for clinical staff to be appropriately trained in the use of PARO in order for the intervention to be effective, with staff in our trial stating that, 'you'd have to have the staff who understood exactly how to use them, and when to use them, and who to use them with' (Moyle, Bramble, Jones \& Murfield, 2016, p. 3). Such training also needs to cover the maintenance of PARO, including how to clean the robot and ensure adherence to infection control procedures. Facility staff involved in our trial commented on the perceived difficulty of keeping PARO’s white fur clean if sharing between residents (Moyle et al., 2016, p. 3), and thus, it is important that staff are supported by management and given time to ensure the 
robot is cleaned after each used, and educated on the importance of doing so from an infection control perspective.

Guidelines advocate the application of multicomponent psychosocial interventions, coupled with individualized support, in managing BPSD (Alzheimer's Association, 2009; National Institute for Health and Care Excellence, 2006, updated 2016; NHMRC Partnership Centre for Dealing with Cognitive and Related Functional Decline in Older People, 2016). A range of psychosocial interventions have demonstrated efficacy for use with this population (Livingston et al., 2014; Oyebode \& Parveen, 2016) - for example music, massage, and animal-assisted therapies - and, as such, there may be an advantage to deliver PARO alongside a suite of other psychosocial interventions. In this sense, residents' can be exposed to the activity alongside a range of other activities, with it becoming part of an individual resident's regular care plan if shown to be beneficial and a source of enjoyment. Such an approach may be the most feasible way in which residents' experience interventions that they find enjoyable, whilst also overcoming the pragmatics of LTC facilities - often limited funding and staff resources - that may prohibit the introduction of individually- and precisely-matched interventions. That said, however, clinicians should be mindful that, as with Margaret - who was highly mobile and constantly walked around the facility restlessness and wandering could make it difficult to engage some residents in any sort of psychosocial intervention. Trying to engage individuals who show no interest in the psychosocial intervention can, in-fact, increase their agitation. Margaret's wandering was not harming herself or others, and she became agitated when interrupted and asked to engage with PARO. In instances such as these, LTC clinicians should consider leaving the resident to walk as they wish, providing that they pose no risk to themselves or others, and continue to offer companionship and opportunities for involvement in activities, as appropriate, and where they show an interest. 


\section{Implications for research}

Comparing a psychosocial intervention against a control group in a RCT design using psychometrically robust assessment scales is the gold standard by which efficacy of interventions are assessed (Bothwell, Greene, Podolsky \& Jones, 2016). Whilst for some, the RCT is considered the only valid research design to establish efficacy (Faraoni \& Schaefer, 2016), recent years have seen increased calls for designs that go beyond the traditional RCT in order to look at wider effectiveness in a real-world context (Alsop, Scott \& Archey, 2016). In our cluster-RCT, we found that, when assessed on our chosen outcome measures (CMAISF and video observation during sessions), PARO had modest but significant effects as a psychosocial intervention to manage the behavioural and psychological signs and symptoms of dementia. However, had a more pragmatic and less rigid RCT been conducted, we may have had different results. For example, had the research design been to introduce PARO when residents asked for it, a more person-centred approach could have been achieved and some residents may have participated in the session rather than refuse. However, in the case of Margaret, her restlessness and wandering are likely to have hindered her engagement in most intervention activities. It is also important to recognise that behavioural and psychological improvement after involvement in an intervention is not always reflected in the chosen assessment scales, and thus, we need to look beyond these for an overall evaluation of intervention effectiveness. Facility staff and family members of residents involved in our study perceived PARO favourably for the person with dementia, noting effects in terms of reducing agitation and vocalisation, increasing alertness, improving mood, and increasing opportunities for communication (Moyle et al., 2016; Moyle, Bramble, Jones \& Murfield, Accepted for publication). These findings are important to consider alongside the main primary outcomes, and we advocate the use of innovative trial designs and methods in future research endeavours to better assess clinical and statistical effectiveness, (e.g., proposed 
design hybrids that combine randomized, pragmatic, and observational trials (Alsop et al., 2016; Faraoni \& Schaefer, 2016; Relton, Torgerson, O'Cathain \& Nicholl, 2010).

\section{Issue 2: Same individual, difference response - the need for continual assessment and review}

Consistent and ongoing monitoring of the person with dementia's response to any intervention should form a key part of care planning. As described for participant 3 (Jessie) within our cluster-RCT, we found that responses to PARO varied considerably for some individuals depending on their mood.

Participant 3 - Different responses on different days: During the video recording of a session with PARO in week 1, Jessie appeared emotionless, displaying neither a positive nor a negative response to PARO. During this session, PARO sat on a table, which Jessie sat at with other residents, facing her. Whilst Jessie sporadically stroked PARO at various points throughout the session, touching PARO's back and flippers, she was mostly unengaged with PARO and tended to stare ahead instead. In week 5, however, Jessie displayed high-levels of agitation, both before and during the session. Appearing unhappy, with a negative facial affect, Jessie repeatedly displayed restless repetitive movements (i.e., pushing PARO around the table), and made numerous attempts to stand-up and move away from the communal table she sat at with other residents. Jessie's attempts to push PARO away resulted in PARO falling from the table onto her lap. She struggled to hold PARO in her arms and change it from its vertical face-down position in her lap. Jessie repeatedly tried to place PARO back on the table and, after appearing unable to lift PARO, Jessie was assisted by the observing RA who put PARO back on the table facing her. During her session with PARO in week 10, Jessie's response to PARO was markedly different from previous weeks. Greeting the RA 
with a warm smile, Jessie welcomed PARO onto her lap and held it with both hands. She showed no signs of agitation towards PARO and happily sat with PARO, stroking and holding it, for the duration of the session.

\section{Implications for practice}

As the described example highlights, it is important not to assume that, because a person with cognitive impairment liked the intervention today, that they will enjoy it tomorrow. As such, before every psychosocial intervention session, clinicians would benefit from undertaking a brief assessment of a number of factors to determine the appropriateness of the intervention for the individual at that particular time. This should include an assessment of the person with dementia’s cognition, agitation, and health, their environment, and interpersonal factors, such as the potential for delirium and adverse effect of medications. Whilst we did not assess cognitive changes over the course of our study, the trajectory of dementia and associated deterioration, including physical frailty, means that the effectiveness of psychosocial interventions can change considerably for an individual over time. However, the application of psychosocial interventions during all stages of dementia remains important, and guidelines continue to advocate their use as a first-line treatment alongside the pharmacological regimen in place (Alzheimer's Association, 2009; National Institute for Health and Care Excellence, 2006, updated 2016; NHMRC Partnership Centre for Dealing with Cognitive and Related Functional Decline in Older People, 2016).

\section{Implications for research}

The described example highlights the issue of response variability for this population, which can be reflective of the characteristic fluctuations in mood and cognisance rather than a response to the intervention itself. Such variability calls into question the use of only a few 
primary outcome assessment time-points in research with this population. To ensure a more balanced assessment of intervention effectiveness, more frequent assessments should be considered. Additionally, researchers should also consider establishing a baseline assessment of behaviour, as was done in our cluster-RCT, whereby participants were recorded for 15mins before they were given the PARO, and then 15-mins with the PARO, at the assessment weeks of 1,5,10, and 15. By taking these pre-and during intervention assessments, the behaviour of the resident immediately preceding the intervention is known and can be accounted for in the analyses.

\section{Issue 3: The ethics of giving and retrieving PARO}

Undertaking any psychosocial intervention in clinical practice or research with a vulnerable population requires a careful benefit/risk assessment and a consideration of possible ethical issues (Slaughter, Cole, Jennings \& Reimer, 2007). This is particularly important when the intervention involves an object that residents can become attached to and feel a sense of ownership towards. In the following examples, we present two responses to PARO that raise important ethical considerations about the giving and retrieval of a psychosocial intervention, such as PARO.

Participant 4 - Possessiveness and attachment to PARO: Betty was sitting watching television with a small group of female residents in the communal lounge area when PARO was introduced to her during a session in week 5. Although displaying a flat facial affect, Betty was immediately engaged with PARO, continuously looking at PARO and stroking its back. Another resident, Mary, sat to her right, and also showed an interest in PARO by persistently leaning over to stroke PARO whilst it sat on Betty’s lap. Betty permitted this interaction initially but, after approximately six minutes, she became agitated and tried to 
push Mary's hand away, saying 'I don't think you should be doing that' and then, a minute later, 'I don’t like it’ when Mary again tried to stroke PARO. Mary did not react to Betty’s dismissals and, when care staff offered Mary afternoon tea, she was distracted and left Betty to interact with PARO on her own for the remainder of the time. When the session was brought to an end by the RA, Betty became visibly distressed, leaning forward from her chair and saying to the RA 'I'm not liking you' and then 'no, why do you have to take him away while he’s here...he’s happy with me.' Betty did not struggle or try to hold onto PARO when it was removed from her lap, but watched with an unchanged flat facial affect until the RA left with PARO. During other videoed sessions with PARO in weeks 1 and 10, Betty did not display signs of possessiveness or attachment to PARO and, in-fact, during both sessions, she asked for PARO to be taken back by the RA.

Participant 5 - Continuing with PARO when there is no engagement: During a session with PARO in week 5, Eric sat with his wife (a non-resident), who was visiting the facility. Eric displayed a flat facial affect and showed very little engagement with PARO, which he held on his lap whilst sporadically talking to his wife and care staff, or staring ahead. After approximately four minutes into the session, Eric stood from his chair, still holding PARO in his arms, and approached the RA to ask if he could take PARO back. The RA told him to place PARO down on the couch to indicate he was finished participating in the session, but Eric returned to his seat, and sat back down with PARO again on his lap. Eric remained unengaged with PARO. In another session in week 10, Eric displayed similar behaviour, sitting unengaged with PARO on his lap in a chair in his bedroom. After about six minutes, Eric voluntarily stood, placed PARO on his bed, and then returned to his chair. 


\section{Implications for practice}

As illustrated in example 4 (Betty), participants can sometimes form a strong attachment to PARO and, consequently, can become distressed when it is removed from them. This attachment can develop over time or, in Betty’s case, can be apparent after some sessions, and not after others. In clinical practice, consideration should be given to how these issues might be mitigated, perhaps through individually-owned intervention objects that are available for the resident to use as-and-when best for them. That said, however, the current cost of PARO - approximately AUD \$8,500, plus annual maintenance and cleaning costs - is likely to preclude many LTC facilities from purchasing multiple PAROs (Moyle et al., 2016). In such instances, consideration should be given to purchasing multiple plush-toys that, whilst not as effective as PARO due to the absence of robotic features, are more effective than standard facility care (Moyle et al., 2017b).

The issue of continuing an intervention when there is little or no engagement is also an important concern in clinical practice. Clinicians have an important role in facilitating the person with dementia to make their own choices and, ultimately, to have their rights upheld and respected. All members of care staff should have education and training to enable them to support the person with dementia in their choices and assess and determine, when needed, the appropriateness of continuing a psychosocial intervention. It is important that assent to participate is sought from all individuals before the commencement of psychosocial intervention sessions, and that this assent should be monitored throughout the activity. Any changes in the demeanour or behaviour that may suggest the individual's wish to stop the session should be noted and acted on to uphold a right-based approach to care (Slaughter et al., 2007). Limited communication skills, however, can make this assessment difficult, with the potential for demonstrated behaviour to be the individual trying to communicate other emotions aside from withdrawal. It could be argued, therefore, that clinicians need a higher 
than normal interpretative standard of what withdrawal may look like for that individual, so that interventions are not stopped too early, and clinicians do not 'give-up' offering the intervention too soon.

\section{Implications for research}

In terms of future research, more work is needed into how long PARO should be left with the older person with dementia. Based on our pilot research, we empirically-derived that intervention sessions with PARO should be with one individual for 15 mins, three times a week, over a 10-week period (Moyle et al., 2013). However, as participants 4 and 5 illustrate, for some individuals the sessions were too short, whereas for other individuals the sessions were too long. Within a RCT design, a standardised and equitable delivery of the psychosocial intervention is paramount to minimise the influence of outside confounding effects. Yet doing so raises important questions about achieving protocol-adherence whilst also upholding participants' freedom of choice. Recently, it has been proposed that a RCT design may not be best suited to use with a dementia population, principally because of its multifactorial, complex, and progressive nature (Ritchie, Terrera \& Quinn, 2015). More pragmatic-based designs allow participants greater freedom of choice (Ware \& Hamel, 2011), and this design may be one possible next step for researchers seeking greater understanding of PARO's effectiveness in routine clinical practice. Research efforts would also benefit from exploring intervention dose as, in our study, a more frequent and longer intervention may have produced a different response over time. However, in designing trials over a longer time-frame, researchers should be mindful that unrestricted access to object-based psychosocial interventions - as seen in research with doll therapy - could sometimes result in participant over-engagement that can lead to emotional and physical fatigue (Mitchell \& Templeton, 2014). 


\section{Conclusion}

The participants we describe within this paper highlight that one approach to reducing BPSD and improving quality of life does not fit all, and that there is considerable variation in responses to PARO both between and within individuals. Complementing the main quantitative results that showed PARO was a feasible and effective intervention, with modest but significant effects these critical reflections provide a more in-depth exploration by looking at individual responses to PARO that would not be revealed by classic quantitative data analytic techniques. The aim is that the issues presented can inform the planning and delivery of psychosocial interventions with PARO in real LTC settings, as well as future research endeavours into PARO’s effectiveness. 


\section{References}

Alsop, J., Scott, M., \& Archey, W. (2016). The mixed randomized trial: Combining randomized, pragmatic and observational clinical trial designs. Journal of Comparative Effectiveness Research, 5(6), 569-579. doi:10.2217/cer-2016-0034

Alzheimer's Association. (2009). Dementia care practice recommendations for assisted living residences and nursing homes. Retrieved from http://www.alz.org/national/documents/brochure_DCPRphases1n2.pdf

Alzheimer's Association. (2016). 2016 Alzheimer's disease facts and figures. Alzheimer's \& Dementia, 12(4), 459-509. doi:10.1016/j.jalz.2016.03.001

Australian Institute of Health and Welfare. (2012a). Dementia in Australia. Cat. no. AGE 70. Retrieved from Canberra: http://www.aihw.gov.au/publication$\underline{\text { detail/?id }=10737422958}$

Australian Institute of Health and Welfare. (2012b). Residential aged care in Australia 201011: A statistical overview. Cat. no. AGE 68. Retrieved from Canberra: http://www.aihw.gov.au/publication-detail/?id=10737422821

Bemelmans, R., Gelderblom, G. J., Jonker, P., \& de Witte, L. (2012). Socially assistive robots in elderly care: A systematic review into effects and effectiveness. Journal of the American Medical Directors Association, 13(2), 114-120.e111. doi:10.1016/j.jamda.2010.10.002

Bothwell, L. E., Greene, J. A., Podolsky, S. H., \& Jones, D. S. (2016). Assessing the gold standard — Lessons from the history of RCTs. New England Journal of Medicine, 374(22), 2175-2181. doi:10.1056/NEJMms1604593

Faraoni, D., \& Schaefer, S. T. (2016). Randomized controlled trials vs. observational studies: Why not just live together? BMC Anesthesiology, 16(1), 102. doi:10.1186/s12871016-0265-3 
Finkel, S. I., \& Burns, A. (1999). BPSD Consensus Statement, International Psychogeriatric Association.

Gaugler, J. E., Wall, M. M., Kane, R. L., Menk, J. S., Sarsour, K., Johnston, J. A., . . . Newcomer, R. (2010). The effects of incident and persistent behavioral problems on change in caregiver burden and nursing home admission of persons with dementia. Medical Care, 48(10), 875-883. doi:10.1097/MLR.0b013e3181ec557b

Gaugler, J. E., Yu, F., Davila, H. W., \& Shippee, T. (2014). Alzheimer's disease and nursing homes. Health Affairs, 33(4), 650-657. doi:10.1377/hlthaff.2013.1268

Gibson, G., Timlin, A., Curran, S., \& Wattis, J. (2004). The scope for qualitative methods in research and clinical trials in dementia. Age and Ageing, 33(4), 422-426. doi:10.1093/ageing/afh136

Jones, C., Sung, B., \& Moyle, W. (2015). Assessing engagement in people with dementia: A new approach to assessment using video analysis. Archives of Psychiatric Nursing, 29(6), 377-382. doi:10.1016/j.apnu.2015.06.019

Jøranson, N., Pedersen, I., Rokstad, A. M. M., \& Ihlebæk, C. (2015). Effects on symptoms of agitation and depression in persons with dementia participating in robot-assisted activity: A cluster-randomized controlled trial. Journal of the American Medical Directors Association, 16(10), 867-873. doi:10.1016/j.jamda.2015.05.002

Klapwijk, M. S., Caljouw, M. A., Pieper, M. J., van der Steen, J. T., \& Achterberg, W. P. (2016). Characteristics associated with quality of life in long-term care residents with dementia: A cross-sectional study. Dementia and Geriatric Cognitive Disorders, 42(3-4), 186-197. doi:10.1159/000448806

Lawrence, V., Fossey, J., Ballard, C., Moniz-Cook, E., \& Murray, J. (2012). Improving quality of life for people with dementia in care homes: Making psychosocial 
interventions work. The British Journal of Psychiatry, 201(5), 344-351. doi:10.1192/bjp.bp.111.101402

Lewin, S., Glenton, C., \& Oxman, A. D. (2009). Use of qualitative methods alongside randomised controlled trials of complex healthcare interventions: Methodological study. BMJ, 339, b3496. doi:10.1136/bmj.b3496

Livingston, G., Kelly, L., Lewis-Holmes, E., Baio, G., Morris, S., Patel, N., . . Cooper, C. (2014). Non-pharmacological interventions for agitation in dementia: Systematic review of randomised controlled trials. The British Journal of Psychiatry, 205(6), 436-442. doi:10.1192/bjp.bp.113.141119

Mitchell, G., \& Templeton, M. (2014). Ethical considerations of doll therapy for people with dementia. Nursing Ethics, 21(6), 720-730. doi:10.1177/0969733013518447

Mordoch, E., Osterreicher, A., Guse, L., Roger, K., \& Thompson, G. (2013). Use of social commitment robots in the care of elderly people with dementia: A literature review. Maturitas, 74(1), 14-20. doi:10.1016/j.maturitas.2012.10.015

Moyle, W., Beattie, E., Draper, B., Shum, D., Thalib, L., Jones, C., . . Mervin, C. (2015). Effect of an interactive therapeutic robotic animal on engagement, mood states, agitation and psychotropic drug use in people with dementia: A cluster-randomised controlled trial protocol. BMJ Open, 5(8), e009097. doi:10.1136/bmjopen-2015009097

Moyle, W., Bramble, M., Jones, C., \& Murfield, J. (2016). Care staff perceptions of a social robot called Paro and a look-alike Plush Toy: A descriptive qualitative approach. Aging \& Mental Health,, 1-6. doi:10.1080/13607863.2016.1262820

Moyle, W., Bramble, M., Jones, C., \& Murfield, J. (Accepted for publication). 'She had a Smile on her Face as Wide as the Great Australian Bite': A Qualitative Examination of Family Perceptions of a Therapeutic Robot and a Plush Toy. The Gerontologist,. 
Moyle, W., Cooke, M., Beattie, E., Jones, C., Klein, B., Cook, G., \& Gray, C. (2013).

Exploring the effect of companion robots on emotional expression in older adults with dementia: A pilot randomized controlled trial. Journal of Gerontological Nursing, 39(5), 46-53. doi:10.3928/00989134-20130313-03

Moyle, W., El Saifi, N., Draper, B., Jones, C., Beattie, E., Shum, D., . . S, O. D. (2017a). Pharmacotherapy of Persons with Dementia in Long-Term Care in Australia: A Descriptive Audit of Central Nervous System Medications. Current Drug Safety,. doi:10.2174/1574886312666170209113203

Moyle, W., Jones, C. J., Murfield, J. E., Thalib, L., Beattie, E. R. A., Shum, D. K. H., . . Draper, B. M. (2017b). Use of a Robotic Seal as a Therapeutic Tool to Improve Dementia Symptoms: A Cluster-Randomized Controlled Trial. Journal of the American Medical Directors Association, 18(9), 766-773.

doi:10.1016/j.jamda.2017.03.018

National Institute for Health and Care Excellence. (2006, updated 2016). Dementia: Supporting people with dementia and their carers in health and social care. Clinical guideline [CG42]. Retrieved from https://www.nice.org.uk/guidance/cg42

NHMRC Partnership Centre for Dealing with Cognitive and Related Functional Decline in Older People. (2016). Clinical practice guidlines and principles of care for people with dementia: Recommendations. Retrieved from http://sydney.edu.au/medicine/cdpc/documents/resources/CDPC-Dementia-

\section{$\underline{\text { Recommendations_WEB.pdf }}$}

Oyebode, J. R., \& Parveen, S. (2016). Psychosocial interventions for people with dementia: An overview and commentary on recent developments. Dementia,. doi:10.1177/1471301216656096 
Petersen, S., Houston, S., Qin, H., Tague, C., \& Studley, J. (2017). The utilization of robotic pets in dementia care. Journal of Alzheimer's Disease, 55(2), 569-574. doi:10.3233/jad-160703

Porter, C. N., Miller, M. C., Lane, M., Cornman, C., Sarsour, K., \& Kahle-Wrobleski, K. (2016). The influence of caregivers and behavioral and psychological symptoms on nursing home placement of persons with Alzheimer's disease: A matched casecontrol study. SAGE Open Medicine 4, 2050312116661877. doi:10.1177/2050312116661877

Prince, M., Knapp, M., Guerchet, M., McCrone, P., Prina, M., Comas-Herrera, A., . . On behalf of Alzheimer's Society. (2014). Dementia UK: Update. Retrieved from London:

https://www.alzheimers.org.uk/download/downloads/id/2323/dementia_uk_update.pd $\underline{f}$

Relton, C., Torgerson, D., O'Cathain, A., \& Nicholl, J. (2010). Rethinking pragmatic randomised controlled trials: introducing the "cohort multiple randomised controlled trial" design. BMJ, 340, c1066. doi:10.1136/bmj.c1066

Ritchie, C. W., Terrera, G. M., \& Quinn, T. J. (2015). Dementia trials and dementia tribulations: Methodological and analytical challenges in dementia research. Alzheimer's Research \& Therapy, 7(1), 31. doi:10.1186/s13195-015-0113-6

Rowland, J. T., Basic, D., Storey, J. E., \& Conforti, D. A. (2006). The Rowland Universal Dementia Assessment Scale (RUDAS) and the Folstein MMSE in a multicultural cohort of elderly persons. International Psychogeriatrics, 18(1), 111-120. doi:10.1017/s1041610205003133

Seitz, D. P., Gill, S. S., Herrmann, N., Brisbin, S., Rapoport, M. J., Rines, J., . . . Conn, D. K. (2013). Pharmacological treatments for neuropsychiatric symptoms of dementia in 
long-term care: A systematic review. International Psychogeriatrics, 25(2), 185-203. doi:10.1017/S1041610212001627

Slaughter, S., Cole, D., Jennings, E., \& Reimer, M. A. (2007). Consent and assent to participate in research from people with dementia. Nursing Ethics, 14(1), 27-40. doi:10.1177/0969733007071355

Takayanagi, K., Kirita, T., \& Shibata, T. (2014). Comparison of verbal and emotional responses of elderly people with mild/moderate dementia and those with severe dementia in responses to seal robot, Paro. Frontiers in Aging Neuroscience, 6, 257. doi:10.3389/fnagi.2014.00257

Thodberg, K., Sørensen, L. U., Videbech, P. B., Poulsen, P. H., Houbak, B., Damgaard, V., . . . Christensen, J. W. (2016). Behavioral responses of nursing home residents to visits from a person with a dog, a robot seal or a toy cat. Anthrozoös, 29(1), 107-121. doi:10.1080/08927936.2015.1089011

van der Spek, K., Gerritsen, D. L., Smalbrugge, M., Nelissen-Vrancken, M. H. J. M. G., Wetzels, R. B., Smeets, C. H. W., . . Koopmans, R. T. C. M. (2016). Only 10\% of the psychotropic drug use for neuropsychiatric symptoms in patients with dementia is fully appropriate. The PROPER I-study. International Psychogeriatrics, 28(10), 1589-1595. doi:10.1017/S104161021600082X

Ware, J. H., \& Hamel, M. B. (2011). Pragmatic trials - Guides to better patient care? The New England Journal of Medicine, 364(18), 1685-1687. doi:10.1056/NEJMp1103502

Werner, P., Cohen-Mansfield, J., Koroknay, V., \& Braun, J. (1994). The impact of a restraintreduction program on nursing home residents. Geriatric Nursing, 15, 142-146. doi:http://dx.doi.org/10.1016/S0197-4572(09)90040-4 
Zimmerman, S., Williams, C. S., Reed, P. S., Boustani, M., Preisser, J. S., Heck, E., \& Sloane, P. D. (2005). Attitudes, stress, and satisfaction of staff who care for residents with dementia. The Gerontologist, 45 Spec No 1(1), 96-105.

Zuidema, S. U., Derksen, E., Verhey, F. R., \& Koopmans, R. T. (2007). Prevalence of neuropsychiatric symptoms in a large sample of Dutch nursing home patients with dementia. International Journal of Geriatric Psychiatry, 22(7), 632-638. doi:10.1002/gps.1722 


\section{Table and Figure Legends}

Table 1. Profile of described participants at baseline of the cluster-RCT

Figure 1. PARO (version 9). Reproduced with permission from Dr Takanori Shibata, The National Institute of Advanced Industrial Science and Technology (AIST), Japan. 


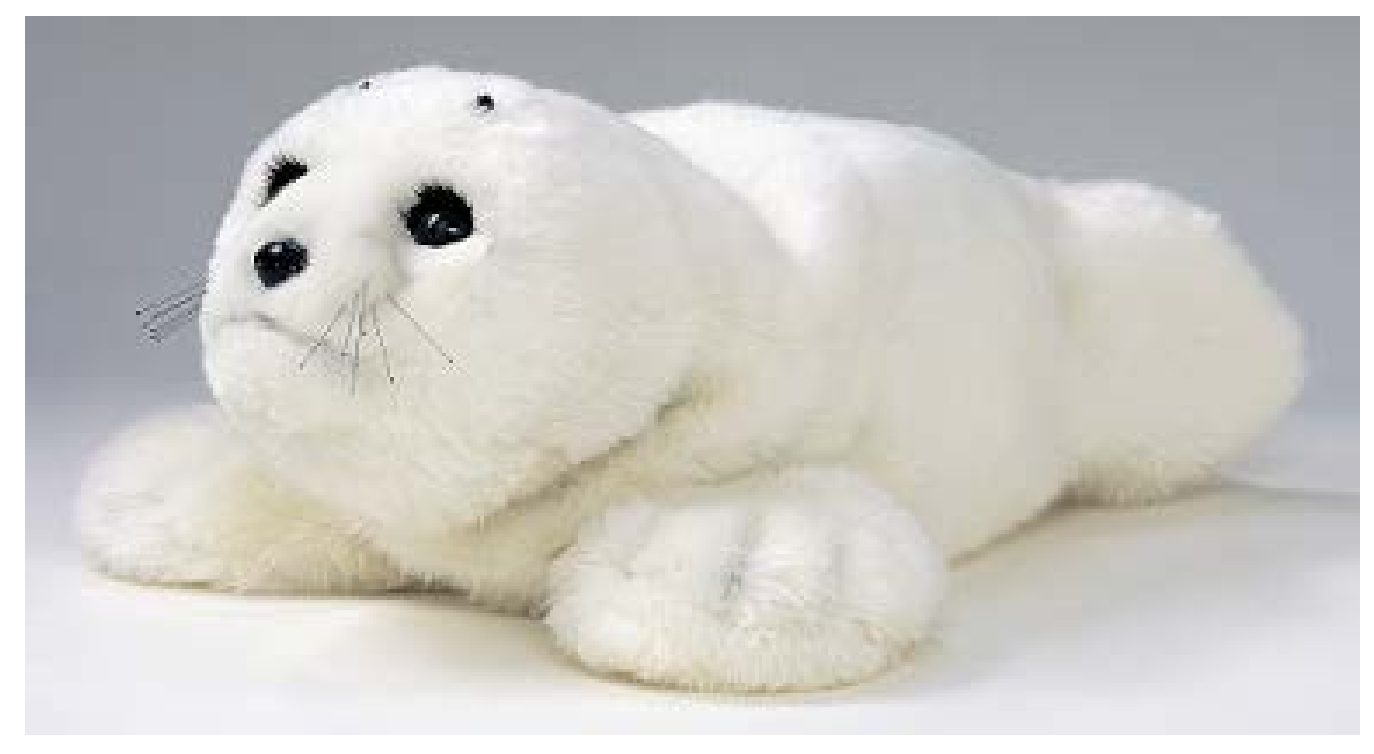

Figure 1. PARO (version 9). Reproduced with permission from Dr Takanori Shibata, The National Institute of Advanced Industrial Science and Technology (AIST), Japan.

[No colour] 
Table 1. Profile of described participants at baseline of the cluster-RCT.

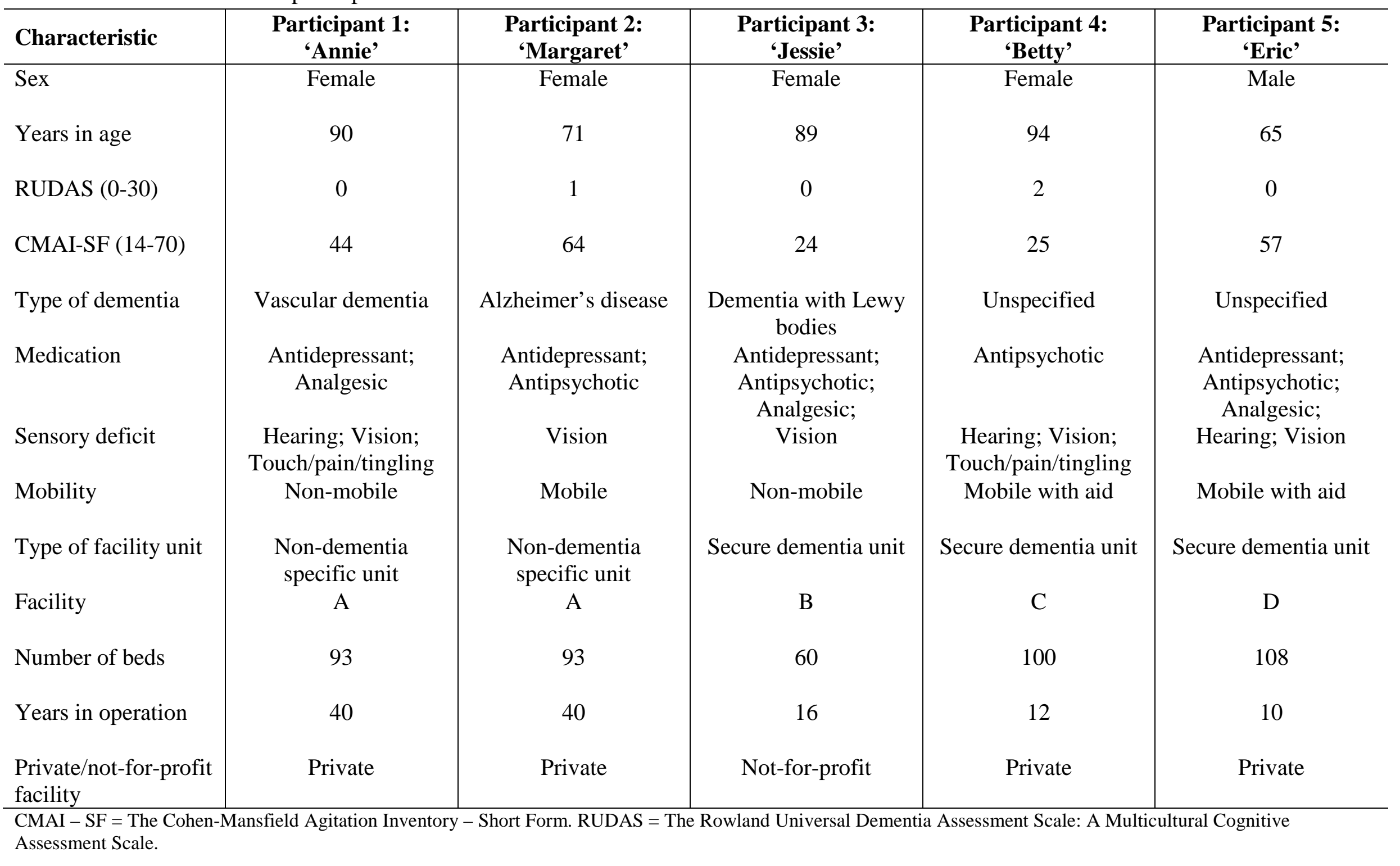

